\title{
Substitutional Semantics and Natural Language Quantification
}

\author{
PETER LUDLOW
}

Columbia University

Substitutional quantification has been proposed in mathematics with the idea of achieving ontological economy'. The idea is simply that a sentence like (1),

(1) Some primes are greater than 7

will be true if and only if $\left(1^{\prime}\right)$ is true.

(1') For some term $t$, replacing ' $x$ ' with $t$ in ' $x$ 18 prime and $x$ is greater than $7^{\prime}$ will result in a true sentence.

This in contrast to the traditional objectual interpretation in which (1) is true if and only if (1\%) is true.

(1') There exists something that satisfies for $x$, ' $x$ is prime and $x$ is greater than 7 '.

(1'') is supposed to commit us to the existence of numbers. (1') is supposed to be free of such ontological commi tment.

In linguistics, it has been observed that substitutional quantification may be useful in cases where quantifiers have wide scope over intentional contexts. For example, it is often held that to get the specific reading of $(2)$,

\section{(2) Polly wants a cow}

(that is, the reading in which Polly has a particular cow in mind), the quantified noun phrase' a cow' will have to take wide scope. If the usual conventions are followed and the indefinite article is treated as an existential quantifier, one gets a structure like $\left(2^{\prime}\right) .^{2}$

\section{$\left(2^{\prime}\right)(3 x: x$ a cow) $[$ Polly wants $x]$}

But of course ( $\left.2^{\prime}\right)$ says that the cow wanted by Polly exists, and such need not be the case. Polly may be 
wishing for Elsie the Borden cow, or the cow that Jumped over the moon.

To account for the undesirable existence ental1ments of sentences like $\left(2^{\prime}\right)$. Fodor has suggested the Introduction of a new quantifier ' $(S x)$ ' which does not connote existence. ' Ioup has agreed with the suggestion, and has gone on to assert that such a quantifier might be thought of as substitutional. On such a theory $\left(2^{\prime}\right)$ would be true if and only if (2'') were true.

(2'') For some term $t$, 't is a cow and Polly
wants $t$ is trues

It seems that substitutional quantification holds some promise both for the philosophy of mathematics and for inguistica. For the purposes of this paper, we w111 focus upon substitutional guantification in inguistics--i.e. on whether natural language quantifiers are substitutional.

The objection to substitutional quantification that is most often cited concerns the problem of unnamed objects. As we shall see, whatever strength this objection may have for formal semantics, it does not apply to the semantics of natural language.

The Idea behind this objection is simply that there are objects which are not named, thus the substitutional interpretation will not be sensitive to the fact that these objects may satisfy certain open sentences. That 1s, under the substitutional interpretation one could never get a proper assignment of truth values for sentences like (3).

(3) Some things are unnamed.

At least not if there are unnamed objects. Obviously. on the substitutional interpretation there would be no names which, when substituted for $x$, could make' $x$ ig unnamed' true.

One necessary condition for this objection to go through is that there are unnamed objects. Prima Eacie there seems to be quite a few unnamed objects. $\frac{\text { c. }}{\bar{J}}$. F. Williams uses the example of unnamed pigeons at Trafalgar Square and argues that the substitutional interpretation assigns the wrong truth value to $(4)^{\circ}$

(4) Something 18 an unnamed pigeon at Trafalgar Square.

Actually, what seems to be a problem with the substitutional interpretation here is more a problem with our carelessness in characterizing valid substitution instances. In most formal substitutional semantics, the metalinguistic quantified expression 'some term $t$ ' ranges over definite descriptions and demonstratives in 
addition to proper names. Thus (4) might be interpreted as true under the substitution of the expression 'the pigeon on the statue of Churchill'.

A more compelling candidate for the status of unnamed object comes from mathematics. There we have learned that each linguistic expression type can be assigned a unique Godel number. If it turns out there are more objects than Godel numbers (1.e. if there are uncountably many objects) then it seems that there must be objects which have no names, definite descriptions, etc. attached to them. As has been well documented elsewhere, there are uncountably many real numbers-hence uncountably many objects in the universe.

While this objection may or may not point out serious limitations in a substitutional account of mathematics, we will find that it has little to do with the question of whether the semantics of natural language is substitutional. (Parenthetically, a number of moves have been proposed which seem to adequately circumvent the view that some numbers must go unnamed in substitutional mathematics. For example, one might follow Henkin and argue that each real number can be named by an Infinite decimal expansion.' The reason why considerations about unnameable objects do not bear upon the semantics of natural language is because they overlook the fact that interpretation is part of our natural endowment. That 18 , one does not learn to interpret quantifiers substitutionally rather than objectually--it is, to borrow a phrase from Chomsky, part of our universal grammar (UG). If one accepts the thesis that the innate principles of UG are genetically determined, then 1 t 18 difficult to see how or why the problem of naming real numbers should have anything to do with the nature of those principles. To think that the 1 ssue does bear upon the semantics of natural language is to suppose elther that our genes foresaw the problem of naming reals or that they evolved in response to the recent discovery that there are such objects. Either alternative seems too absurd for consideration. The point is that issues in the semantics of natural language should be decided by intuitions about sentences encountered on a daily basis--not by sentences about highly technical mathematical objects which are best treated in an artificial language. We cannot overlook the possibllity that natural language breaks down in mathematical discussions of a highly technical nature.

of course, none of this is to say that the interpretation of quantifiers in natural language $1 \mathrm{~g}$ substitutional. It is merely to say that the problem of unnamed objects sheds no light on the guestion.

In fact, there $1 \mathrm{~s}$ some guestion as to whether the substitutional strategy will work for all natural language guantifiers. As we shall see below, it is extremely difficult to give a substitutional account of 
restricted quantifiers. In fact, we will see that an account may be had only by way of massive violence to the ayntas of natural language. guantifiers.

Consider the following sentences with restricted

(5) Exactly one person wrote Waverly.

(6) Seven spies (each with two code names) quit the CIA.

The most natural way of interpreting (5-6) would be as In $\left(5^{\prime}-6^{\prime}\right)$

(5') For exactly one term $t$ ' $t$ is a person and $t$ wrote Waverly' is true.

(6') For exactly 7 terms $t 1, t 2, \ldots, t 7^{\prime}$ 't quit the CIA' is true.

The problem with $\left(5^{\prime}\right)$ and $\left(6^{\prime}\right) 18$ that they will not be aspigned the correct truth value at interpretation. $\left(5^{\prime}\right)$ will be assigned the value false because there are at least two names which will make ' $t$ wrote Waverly' true--'Scott' and 'sir Walter'. And likewise, because each opy has several names, (6') will be assigned the value false even when seven sples did quit the CIA. The problem becomes even more sertous when we recall that definite descriptions and demonstratives may also serve as substitution instances.

At first glance, it seems as though the difficulty fust sketched is easily handled. One way in which it might be circumvented would be to interpret (5-6) as $\left(5^{\prime}-6^{\prime}\right)$

$\left(5^{\prime \prime}\right)$ For some term $t$, 't wrote Waverly and (x) ( $x$ wrote Waverly $\rightarrow x=t)^{\prime}$ is true.

(6'') For seven terms $t 1, t 2, \ldots, t 7$, 't1 is a spy and $t i$ left the CIA and $(x)((x)$ is $a$ spy and $x$ left the CIA $\rightarrow(x=t 1$ v $x=t 2 \vee \ldots v x=t 7))^{\prime}$ is true.

(5') seems to capture what is being said in (5). but $\left.16^{\prime}\right)$ is not sufficient to get (6). The problem with $\left(6^{\prime}\right)$ is that it fails to exclude the possiblity that $t i=t j$. That 18 , there 18 no reason to suppose there 18 not just one spy with 7 names such that that one spy could satisfy $\left(6^{\circ}\right)$. Thus, we might try $\left(6^{\cdots} \cdot\right)$

$\left(6^{\prime \prime}\right)$ Eor seven terms $t 1, t 2, \ldots, t 7$, 't1 is a spy and $t i$ left the CIA and $(x)((x$ is $a$ spy and $x$ left the $C I A) \rightarrow(x=t 1 \vee x=t 2 v \ldots v x=t 7))$ and 
$(t 1 \neq t 2=\ldots \neq t 7)^{\prime}$ is true

If the substitutional treatment of quantifiers like 'seven' is baroque, treatment of so-called non-firstorderizable quantifiers like 'most' is an absolute mess. Consider a sentence like (7),

(7) Most men wear hats.

(7) is a bit troublesome even on an objectual account, for it makes a claim about properties of sets. Namely. it claims that the cardinality of the set of hat wearers 1s greater than the cardinality of non-hat-wearers. Thus, on an objectual account, (7) is treated as $\left(7^{\circ}\right)$.

$\left(7^{\prime}\right) /(x: x$ is a man and $x$ wears a hat $) />/(x: \quad x$ is a man and $x$ doesn' $t$ wear a hat) $/{ }^{\prime}$

A naive way of trying to substitutionally interpret (7). would be along the lines of (7'). For example. $\left(7^{\circ}\right)$.

(7' $) /(x: \quad x$ is a term $t$ and $' t$ is a man and $t$ wears a hat' is true $) />/(x: x$ is a term $t$ and ' $t$ is a man and $t$ doesn' $^{\prime}$ wear a hat' is true)/

But of course, there is no reason to suppose that (7' ') will be true if and only if (7) is true, for there 18 no reason to suppose there are as many names for hat wearers as there are for non-hat-wearers. In fact, given that there are an infinite number of possible descriptions for each hat wearer and non-hat-wearer it is in principle impossible to over determine when $\left(7^{\prime}\right)$ will be true.

The fact of the matter is that on the substitutional interpretation, quantifiers like 'most' are not only non-first-orderizable, they are non-second-orderizable. One must establish the cardinality of sets of sets of terms. (7), for example, would have to be interpreted as $(7 \cdots)$.

$(7 \cdots \cdots) /(x: \quad x$ is a set $s=(t(1), t(2) \ldots t(n))$ where $t(1), t(2) \ldots t(n)$ are terms and for each $t(i), t(j)$ in $s, t(1)=t(j)$ and $t(1)$ is a man and $t(i)$ wears a hat' is true and for all terms $y,\left({ }^{\prime} y=t(1)^{\prime}\right.$ is true $\rightarrow$ $y \in s) />/(x:$ x is a set $s=(t(1), t(2) \ldots t(n))$ where $t(1), t(2), \ldots, t(n)$ are terms and for each $t(1), t(j)$ in $s, t(1)=t(j)$ and $t(1)$ is a man and $t(i)$ doesn' $t$ wear a hat' is true and for all terms $y,($ ' $y=t(1)$ ' is true $\rightarrow$ yes)/

While such maneuvering may be acceptible for formal semantics, it is certainly not acceptible in a theory of the semantice of natural language. 
In order to get representations 11 ke $\left(6^{\prime}\right)$ ) and (7' ') one wolld need some sort of rule or algorithm to generate them from surface structures 11 ke (6) and (7). On the Extended standard Theory of transformational grammar, for example, one would need an additional level of representation (Bay LE') and a rule that would map to LE from LE or some other level of syntactic representation. Whether such a rule could even be constructed 18 questlonable, for it is clear that no $8 \mathrm{im}-$ ple transformation 111 generate $\left(6^{\prime \prime}\right)$ ) from (6). There would have to be a rule that completely rewrites (6). generating from it a structure which bears no syntactic relation to (6). Furthermore, this rule would have to be general enough to work for a large class (preferably all) of the restricted quantifiers. One would not want a different mapping rule for each quantifier. However, it 18 already apparent that one rule is unlikely to generate both $\left.\left(6^{\prime}\right)^{\prime}\right)$ and $\left(7^{\cdots}\right)$.

Following Hornstein and Lightfoot' one can hold there are three conditions of adequacy for a theory of natural language; empirical adequacy, explanatory adeguacy, and ofmplicity. Empiricial adequacy would relate to how well the theory fit the facts--that is, whether it is consistent with the available data. Explanatory adequacy would concern how well the theory explained the logical problem of language acquisition. And simplicity, as we might suppose, is a criterion that favors elegant theorles that are unencumbered by ad hoc rules and principles. It should be apparent by now that even if one can formally describe rules that w111 give an appropriate account of restricted quantiflers--that is, even if one can make such a theory fit the facts (and there is no reason to suppose that one can)--the theory will fail on other conditions.

It 18 perhaps most obvious that such a theory will fall on grounds of simplicity. Not only will there have to be the introduction of a number of new rules and possibly levels of representation to give an account of restricted quantification, there will also be the introduction of new kinds of rules. These new rules will not be simple transformations nor will they merely describe the relations holding between different syntactic components. Rather they will completely rewrite the sentence. One could easily argue that the number of kinds of components in a theory is just as Important as the number of components themselves.

Finally, it should be clear that such a theory would shed iftele light on the logical problem of language acguistion. Surely it would be odd if for every new quantifier that we learned to use, we had to represent it in a completely new manner. Such a result would entail that we do not have a general algorithm for representing and understanding quantifiers, but that we have to learn to represent each of them on a 
case by case basis. And this seems simply devastating for any theory of language acquisition.

\section{NOTES}

*This paper, while short, is the product of the help of many people. An earlier, much more general paper on this topic recelved a number of helpful comments from Charles Parsons and Robert May. A more refined version of the material was presented informally at MIT, where very helpful comments were provided by Jim Higginbotham, Richard Larson, and Dan Finer. Further sharpening of certain points came out of discussion of these 1 ssues with Noam Chomsky and Norbert Hornstein. Finally, I would like to thank the MIT Department of Linguistics and Philosophy for making their facilities avallable to me during my tenure as a Visiting Scholar.

'Eor example, Dale Gottlieb, Ontological Economy: Substitutional quantification and Mathematics, (Oxford: Oxford University Press, 1980).

${ }^{2}$ Noam Chomsky has suggested to me that it is $111 \mathrm{e}-$ gitimate to suppose that natural language guantifiers can be represented as the guantifier $(\exists x)^{\prime}$ found in formal logic. I take it that Chomsky's point is that one should not import too many devices from formal logic, for doing 80 can color how we view natural language. While I think that Chomsky's point is in general a good maxim, it is not so clear that the usual dangers surface here. ' $(3 x)^{\prime} 18$, after all, an undefined symbol, and there is no reason why it could not stand proxy for any component of the syntax. We might just regard it as a happy colncidence that natural 1 anguage guantifiers behave as do formal ones. Chomsky's point may be that natural language guantifiers must interract with pragmatics before they begin to behave like quantifiers in formal semantics. That is, he could be saying that they should be represented like the formal device ' $(3 x)^{\prime}$ ' only at some level LE'. where the gramar interracts with other aspects of human psychology. My intuition 18 that such a view 18 incorrect (see my Columbia University dissertation, forthcoming).

Janet Fodor. The Linguigtic Description of Opaque Contexts, MIT dissertation, 1970. 
"Gentgotte Ioup, "specificlty and the Interpretation of ginntifiers, Linguistics and philosophy, 1. (1977).

'Herp I have used a notational short-cut that w111 be ured throliqhout the rest of the paper. Rather than apeaking of "replacing ' $x$ " wtth $t$ in $x$ is a cow" I have elected to simply say ${ }^{\circ} t$ is a cow'. Those who fint my notational. Il cense troublesome or confusing may want Lo replace my talk of substituting within quotation wilh talk of concetenation. Thus, one could say, "Ent some term $t, t$ 'is a cow" 1s true. Metalinguistic quantifiers are token to be objectual.

'C.J.F. WIlliams, What 18 Existence? (Oxford: Oxford University Press, 1981 , p. $\frac{193 .}{193}$

'L. Henkin, W. N. Smith, V. J. Varineau and M. J. Walsh, Retracing Elementary Mathematics, (New York: Macmillan Co.. (1962). Pp. 390-97.

Charles Parsons has pointed out to me that cardinality may not provide the best way of treating such quantifiers. One of his reasons for harborting suspicion about such an approach is that if quantifiers like 'most' are needed to give an account of mathematics (and thus cardinality), the quantifier will be defined by certain concepts which employ that very guantifier in their own definitions. Whether or not such difficulties are in fact encountered in a treatment of natural language, It 18 clear that any other treatment of 'most' 18 bound to be just as complicated.

Introduction to Hornstein, Norbert and David Lightfoot, eds. Explanation in Lingulstics: The Logical Problem of Language Acquigition, (London: Long- 